\title{
Magnetic resonance imaging-guided biopsies in children
}

Acta Radiologica Open

I0(II) 1-9

(C) The Author(s) 2021

Article reuse guidelines:

sagepub.com/journals-permissions

DOI: $10.1177 / 20584601211053846$

journals.sagepub.com/home/arr

(S)AGE

\author{
Mika Hirvonen' ${ }^{\circledR}$, Juha-Jaakko Sinikumpu², Osmo Tervonen' and \\ Roberto Blanco Sequeiros ${ }^{3}$
}

\begin{abstract}
Background: Magnetic resonance imaging (MRI) is used far less as an imaging-guided method for percutaneous biopsies than computed tomography (CT) and ultrasound (US), despite its imaging benefits, particularly in children.

Purpose: To evaluate the feasibility, accuracy and safety of MRI-guided biopsies in paediatric patient population.

Material and Methods: The retrospective study included 57 consecutive paediatric patients (<I8 years old). A percutaneous core needle biopsy (PCNB) or trephine biopsy was performed in 53 cases, and an additional fine-needle aspiration biopsy (FNAB) in 26 cases. In 4 cases, a stand-alone FNAB was taken. Biopsies were performed with $0.23 \mathrm{~T}$ open and I.5 T closed MRI scanners. Statistical methods used for confidence intervals and $p$-values were Wilson score method and chi-square test.

Results: The overall diagnostic accuracy of histologic biopsy was 0.94 , with sensitivity 0.82 , specificity 1.00 , positive predictive value (PPV) 1.00 and negative predictive value (NPV) 0.92. In histological bone biopsies, diagnostic accuracy was 0.96, with sensitivity 0.86 , specificity I.00, PPV I.00 and NPV 0.94. The FNAB sample diagnosis was associated with the histological diagnosis in $79 \%$ of cases. There were no major primary complications and only a few late complications. After biopsy, $83 \%$ of the children were ambulatory in $6 \mathrm{~h}$. Anti-inflammatory drugs and paracetamol provided satisfactory pain relief in $96 \%$ of the patients after biopsy. Most outpatients (71\%) were discharged from hospital either on the same day or I day later.
\end{abstract}

Conclusion: $\mathrm{MRI}$ is a technically feasible, accurate and safe guidance tool for performing percutaneous biopsies in children.

\section{Keywords}

Magnetic resonance imaging, interventional magnetic resonance imaging, pediatrics, tumour, biopsy

Received 10 December 2020; Accepted 28 September 202I

\section{Introduction}

In the past, open biopsy or surgery has been the preferred method to obtain histopathologic diagnoses of suspicious masses in children. ${ }^{1}$ With image guidance, biopsies and procedures can be performed considerably less invasively than through open surgery. ${ }^{2,3}$ The potential complication risks associated with image-guided biopsies and open surgery do not significantly differ. ${ }^{4}$ For children, despite its
'Department of Radiology, Oulu University Hospital, Oulu, Finland ${ }^{2}$ Department of Paediatric Surgery and Orthopaedics, PEDEGO Research Center, Oulu University Hospital and Oulu University, Oulu, Finland ${ }^{3}$ Department of Radiology, Turku University Hospital, Turku, Finland

\section{Corresponding author:}

Mika Hirvonen, Department of Radiology, Oulu University Hospital, Kajaanintie 50, P.O. Box 50, 90029 OYS, Oulu 90220 Finland.

Email: Mika.Hirvonen@ppshp.fi 
imaging benefits, magnetic resonance imaging (MRI) is far less used for image-guided percutaneous biopsies or as an intervention method than computed tomography (CT) and ultrasound (US). The advantages of MRI are excellent spatial and contrast resolution especially in soft tissue, good flow detection, multiplanar imaging and no radiation. MRI is well suited for the planning, guidance and monitoring of diagnostic and therapeutic procedures., ${ }^{2,5}$ For children, ALARA (As Low As Reasonably Achievable) is a crucial principle that aims to minimise radiation exposure. One main limitation of interventional MRI is the powerful magnetic field involved; all instruments and equipment must be compatible with the MRI environment. Other limitations are a longer procedure time due to the relatively long imaging time, higher costs and the limited availability of MRI scanners. Moreover, a closed bore MRI does not allow direct patient access during imaging. Implants can be contraindications and cause artefacts but are rarely a problem with children. ${ }^{2,5,6}$

With children, most studies on image-guided biopsies have focused on CT and US guidance. ${ }^{7-21}$ There are few publications on MRI-guided biopsies in children and published patient series are small (range 1-13 cases). ${ }^{4,22-25}$ The accuracy of MRI-guided bone biopsies correlates with open and CT-guided biopsies. ${ }^{26}$ However, to our knowledge, there is only one study in adults that reported accuracy of MRI-guided bone biopsies in a larger population. ${ }^{27}$

The purpose of this study was to evaluate the feasibility, accuracy and safety of MRI-guided biopsies in paediatric patient population.

\section{Materials and Methods}

\section{Patients}

This retrospective study was approved by the institutional ethical board and consisted of 57 consecutive paediatric MRI-guided percutaneous biopsies performed from May 1999 to November 2016. Inclusion criteria were: ${ }^{(1)}$ age less than 18 years; ${ }^{(2)}$ unconfirmed and possibly neoplastic bone or soft tissue lesion detected in a previous radiograph, US, CT, MRI, bone scan or combination of these modalities. Eight of 57 children had a known primary malignancy. Of these 8 , seven were symptomless and with a stable followup phase. The patients' age ranged from 3 months to 17 years (mean 12.2 years), with 32 girls and 25 boys. In 18 of 57 cases, the biopsy was taken during an MRI-guided therapeutic procedure of a bone lesion to ensure benignity. Patients with implants or medical hardware preventing the use of MRI as a guidance method were excluded.

The anatomical locations of the target lesions are shown in Table 1. In $53(96.2 \%)$ cases, a percutaneous core needle biopsy (PCNB) or trephine biopsy was performed. In 26 (45.6\%) cases, a fine-needle aspiration biopsy (FNAB) was combined to histological biopsy. In $4(7.0 \%)$ cases, a stand-alone FNAB was taken. In 20 cases, a microbiological culture sample was combined due to the nonspecific nature of the imaging findings.

\section{Equipment}

A $0.23 \mathrm{~T}$ open and a $1.5 \mathrm{~T}$ closed MRI scanner were used. Both scanners enabled real-time or near-real-time imaging during the biopsy and procedural control was achieved by taking images before and after moving the instrument.

Four different calibres of MRI-compatible trephine biopsy sets $(2,3,4$ and $6 \mathrm{~mm})$ were used in bone biopsies. In recent years, a $4 \mathrm{~mm}$ set has been standard (Figure 1). A soft tissue PCNB was performed with a 12- to 18-gauge (G) needle.

FNAB was performed through an MRI-compatible coaxial needle set with MRI-compatible 18- to 20-G needles. Part of the FNABs were combined with a trephine biopsy/ PCNB; here, a semi-automated MRI-compatible true-cut needle and MRI-compatible coaxial needle were used.

\section{Procedure}

The biopsy route was planned using the primary MR images $(n=55)$ or imaging during the procedure $(n=2)$. The most viable appearing or enhancing part of the lesion was selected as the target. In the low-field (open bore) MRI, T1-and T2weighted fast spin echo (FSE)/turbo spin echo (TSE), field echo (FE)/fast field echo (FFE) and True-FISP gradient echo sequences were used to localise the target and the biopsy set (Table 2). In the high-field (closed bore) MRI, T1 and T2 TSE and T2 Haste sequences were used (Table 3 ).

Table I. Anatomical locations of target lesions.

\begin{tabular}{|c|c|c|}
\hline Anatomical sites of bone lesions & Frequency & Percent \\
\hline Femur & 20 & 44.4 \\
\hline Tibia & 8 & 17.8 \\
\hline Spine & 4 & 8.9 \\
\hline Ankle/foot & 4 & 8.9 \\
\hline Pelvis & 4 & 8.9 \\
\hline Upper limb & 4 & 8.9 \\
\hline Ossification centre of patella & 1 & 2.2 \\
\hline Total & 45 & 100.0 \\
\hline Anatomical sites of soft tissue lesions & Frequency & Percent \\
\hline Internal organ ${ }^{\mathrm{a}}$ & 3 & 25.0 \\
\hline Disc/spine & 3 & 25.0 \\
\hline Other soft tissue ${ }^{b}$ & 6 & 50.0 \\
\hline Total & 12 & 100.0 \\
\hline
\end{tabular}

${ }^{\mathrm{a}} \mathrm{A}$ liver lesion, two pancreas lesions.

${ }^{\mathrm{b}} \mathrm{A}$ synovia of the knee, a popliteal lymph node, a tumour of the nape, a tumour of the piriformis muscle, a parapedicular soft tissue lesion, a soft tissue thickening around the periosteum. 


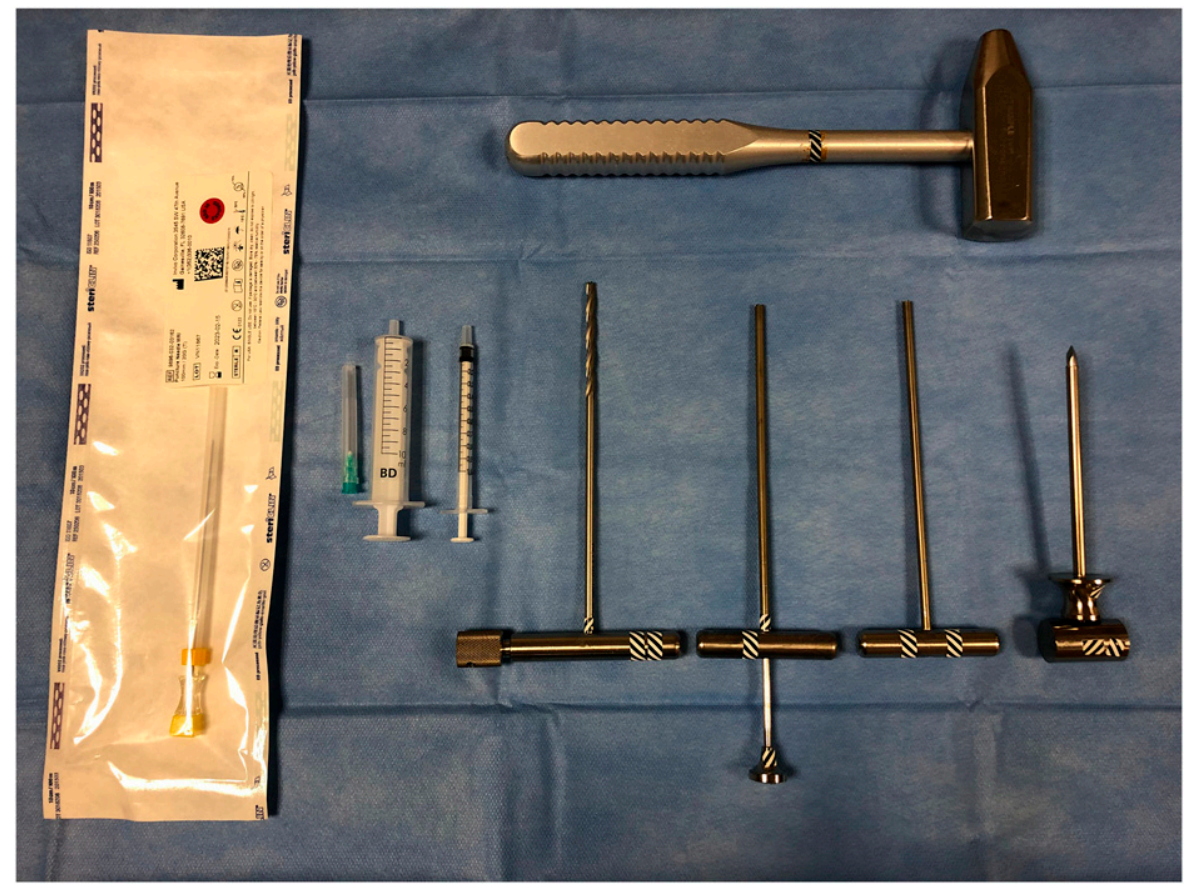

Figure I. Instrumentation for MRI-guided bone biopsy (4 mm set). From left to right: $20-\mathrm{G}$ puncture needle, needle and syringe (2I G/ $10 \mathrm{~mL}$ ) for local anaesthetic, syringe for gadolinium-contrast agent (for skin entry point marking), manual spiral drill, two different kinds of cavity drill (one with ejector), trocar and mandrin for bone entry and top crosswise, a hammer. Note: MRI: magnetic resonance imaging.

Table 2. Open MR imaging sequences used.

\begin{tabular}{lllllllll}
\hline Sequence & TE $(\mathrm{ms})$ & TR $(\mathrm{ms})$ & $\begin{array}{l}\text { Slice thickness } \\
(\mathrm{mm})\end{array}$ & $\begin{array}{l}\text { Slice interval } \\
(\mathrm{mm})\end{array}$ & FOV & Matrix & $\begin{array}{l}\text { No. of } \\
\text { slices }\end{array}$ & $\begin{array}{l}\text { Acquisition } \\
\text { time }\end{array}$ \\
\hline TI FE/FFE & 7 & $60-95$ & $7-8$ & $5-10$ & $200-380 \times 200-380$ & $270-300 \times 270-300$ & $3-5$ & $7.9-12.5$ \\
TI FSE/TSE & $16-18$ & $400-420$ & $3.5-7$ & $4-8$ & $150-380 \times 150-380$ & $288-324 \times 288-324$ & $5-16$ & 14.9 \\
T2 FSE/TSE & $100-150$ & $2000-4200$ & $3-7$ & $3.5-8$ & $160-380 \times 160-380$ & $160-256 \times 160-256$ & $5-9$ & $19.4-23.3$ \\
TRUE-FISP & $3.8-5.0$ & $7.6-9.0$ & $5-10$ & $3-10$ & $200-380 \times 200-380$ & $160-256 \times 160-256$ & $1-12$ & $1.1-9.8$ \\
\hline
\end{tabular}

Table 3. Closed MR imaging sequences used.

\begin{tabular}{lllllllll}
\hline Sequence & TE $(\mathrm{ms})$ & TR $(\mathrm{ms})$ & $\begin{array}{l}\text { Slice thickness } \\
(\mathrm{mm})\end{array}$ & $\begin{array}{l}\text { Slice interval } \\
(\mathrm{mm})\end{array}$ & FOV & Matrix & $\begin{array}{l}\text { No. of } \\
\text { slices }\end{array}$ & $\begin{array}{l}\text { Acquisition } \\
\text { time }(\mathrm{s})\end{array}$ \\
\hline TI TSE & 9.9 & $291-484$ & $3-5$ & $3.9-6.5$ & $150-250 \times 150-250$ & $256-384 \times 256-384$ & $3-10$ & $6.2-18.5$ \\
T2 TSE & 78 & $1000-2304$ & $3.5-5$ & $3.9-5.5$ & $180-260 \times 180-260$ & $384-512 \times 384-512$ & $3-8$ & $5.0-11.5$ \\
T2 HASTE & 108 & 1000 & 6 & 7.2 & $203-300 \times 203-300$ & $156-192 \times 156-192$ & 10 & 3.6 \\
\hline
\end{tabular}

Before the biopsy, the entry point on the skin was localised. Antiseptic skin preparation, skin coverage and safety issues were completed. The biopsy route and the entry point were determined by placing the tracker-mounted instrument $^{26,28}$ or gadolinium-filled syringe close to the skin $^{2}$ and evaluating the marker, target and route from consecutive images before entry. Local anaesthetic (usually lidocaine) was infiltrated intra- and subcutaneously. Through a skin incision, the coaxial needle was advanced to the region of interest with the help of the instrument-generated susceptibility artefact (Figure 2). The trocar and mandrin were removed from the coaxial cannula after target contact and samples were collected. If necessary, a manual MRI-compatible spiral drill (In Vivo Corporation, Gainesville, USA) was used in deeper 
targets for bone channelling before the actual biopsy. Soft tissue lesions were biopsied with a semi-automated MRIcompatible side-cutting biopsy gun (In Vivo Corporation, Gainesville, USA and Somatex, Berlin, Germany). Bone biopsies were taken with a cylindrical trephine (In Vivo Corporation, Gainesville, USA). The biopsy was repeated if the sample seemed macroscopically insufficient. In cases of PCNB or trephine biopsy, the combined FNAB was aspirated through the cannula after the initial biopsy. A standalone FNAB was taken in a similar manner through a smaller diameter coaxial needle.

There was no pre-established practice in the use of prophylactic antibiotics. Altogether, $14(24.6 \%)$ cases received prophylactic antibiotics; all were bone biopsy cases. No contrast agent was used during the biopsies.

The choice of anaesthesia method was made by the anaesthesiologist in collaboration with a radiologist. All but two procedures were performed under general anaesthesia; there was one spinal and one local anaesthesia. All biopsies were performed by experienced interventional radiologists.

\section{Outcome variables}

Feasibility was evaluated by recognising any technical challenges during biopsy and by analysis of the needle and procedure time.

Biopsy accuracy was determined by using the histopathological diagnosis as a reference for the final diagnosis. The final diagnosis was based for all patients on imaging findings, MRIguided biopsy and possible re-biopsy results, possible surgical biopsies and extirpations, follow-ups and clinical findings. The diagnostic relevance of the MRI-guided biopsy result was compared to the final diagnosis after follow-up. Based on the follow-up, the biopsy results were classified as true positive (TP), true negative (TN), false positive (FP) or false negative (FN). Considering all the biopsies, the minimum follow-up was 3 months (range 1 month-11 years). All samples were classified as either diagnostic or non-diagnostic, and a specific histopathological diagnosis was set by a pathologist. In cases where histopathology of primary or repeated biopsies showed no signs of malignancy or infection and remained partially nonspecific despite the technical success of the biopsy procedure and sampling, the biopsy results were classified as diagnostic for a benign lesion, as long as no evidence of malignancy or infection was found during the follow-up or surgery.

Technical observations took into account the mean lesion diameter, size of the needle tip artefact, number of passages and post-procedural recovery. The need for additional biopsies was also reported.

Safety evaluation was based on adverse events.

\section{Statistical method}

The data of diagnostic performance are given as numbers of TP, TN, FP and FN. Diagnostic accuracy statistics (sensitivity, specificity, positive predictive value (PPV), negative predictive value (NPV), diagnostic accuracy (DA)) with their 95\% confidence intervals were calculated for each finding. The confidence intervals were calculated using Wilson score method without continuity correction. The $p$-values were calculated with chi-square test.

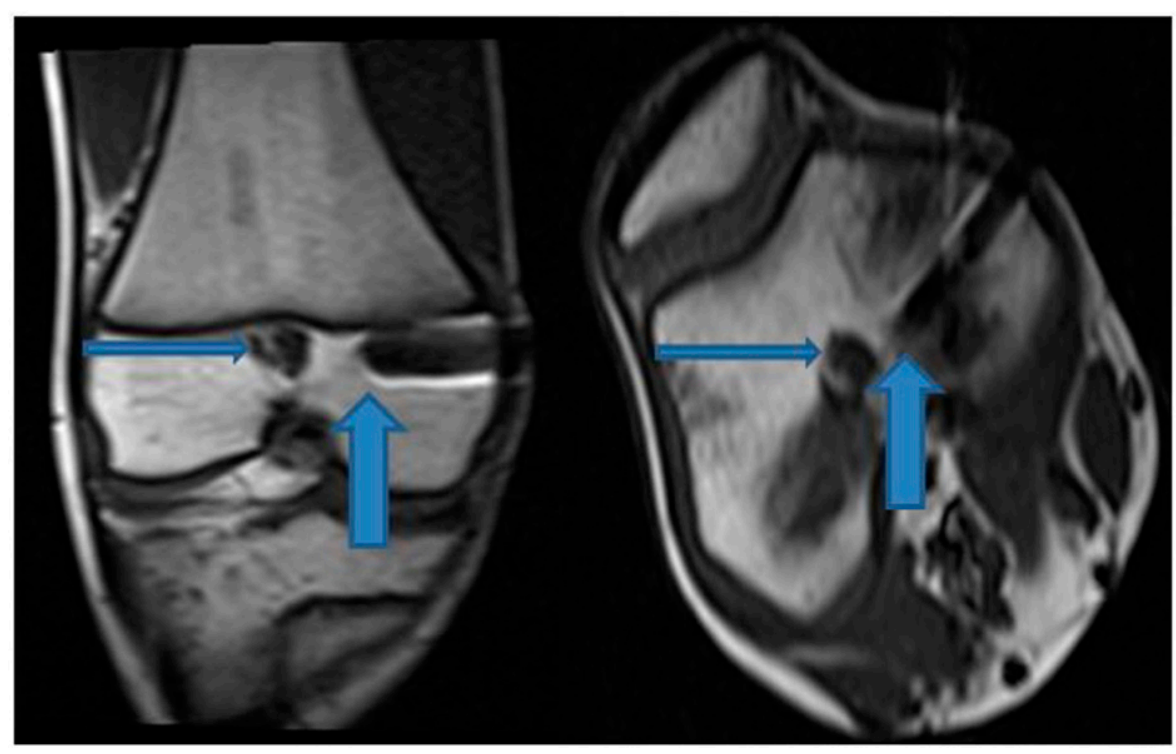

Figure 2. MRI-guided biopsy of a well-defined lesion located in the distal femur just below the epiphyseal plate. On the left side, coronal plane (TI TSE) and on the right side, axial plane (TI TSE). The thicker arrow points to the tip of the biopsy needle (including trocar and mandrin) and the thinner arrow to the biopsy target, which is $1.5 \mathrm{~cm}$ in diameter. The lesion was an enchondroma. Note: MRI: magnetic resonance imaging; TSE: turbo spin echo. 


\section{Results}

\section{Feasibility}

The histological sampling was successful in every case, but in eight of $53(15.1 \%)$ cases, there were some technical challenges. In two soft tissue lesions, technical challenges were related to the small size and rubber-like matrix of the lesion. In five bone lesions, the sclerotic hardness of a lesion, mild bleeding from the drill tract, poor distinctiveness of the nidus in a sclerotic lesion, limited steering properties of the drill regarding to the target and shape of the focus (patella) caused some technical challenges. In one case, a small piece of drill edge was lodged inside the bone lesion but was easily removed with the extension of the tract. In all $30 \mathrm{FNAB}$ cases, the sampling was successful, although the small size of the lesion in one case caused a technical challenge.

Technical challenges were associated with the needle and procedural times in PCNBs and trephine biopsies (Table 4). The needle time was 2.4-fold and the procedural time 2-fold longer in cases with technical challenges compared to cases without technical issues. The needle time varied from 33 to $114 \mathrm{~min}$ in the group with technical challenges, and from 4 to $141 \mathrm{~min}$ in the technically non-problematic group. The procedural time range in the respective groups varied from 40 to $125 \mathrm{~min}$ versus from 7 to $164 \mathrm{~min}$. In two of the 8 cases with technical challenges, procedural MRI images were not stored in a digital archive.

Thirty FNABs were taken and in 26 of them, FNAB was combined with histological sampling. The needle time range was 10-40 min (mean $19.5 \mathrm{~min}, \mathrm{SD} \pm 14.0 \mathrm{~min}$ ) when a stand-alone FNAB was performed, and 4-141 min (mean $49.7 \mathrm{~min}, \mathrm{SD} \pm 34.2 \mathrm{~min}$ ) when FNAB was combined. The procedural time range in these groups was 32-61 min (mean $40.0 \mathrm{~min}, \mathrm{SD} \pm 14.0 \mathrm{~min}$ ) and 7-164 min (mean $66.1 \mathrm{~min}$, $\mathrm{SD} \pm 38.0 \mathrm{~min})$, respectively.

\section{Accuracy}

Fifty-one of 53 (96.2\%) PCNBs and trephine biopsies were diagnostic. Twenty-four of $30(80.0 \%)$ FNABs were diagnostic. Two of four samples were non-diagnostic in cases where only FNAB was taken. In two of 26 cases, where both $\mathrm{PCNB} /$ trephine biopsy and FNAB were taken, both histological and cytological samples were non-diagnostic, both soft tissue lesions. In 19 of the remaining $24(79.2 \%)$ cases, the histology and cytology corresponded. In two of 5 cases where there was no correspondence, the PCNB/trephine biopsy was diagnostic, and the FNAB was undiagnostic.

The accuracy and overall diagnostic performance are summarised in Table 5. Histological bone biopsy had better sensitivity than cytological bone biopsy ( $86 \%$ vs $43 \%, p=$ $0.120)$. The results were similar for accuracy ( $96 \%$ vs $75 \%$, $p=0.036)$ and NPV (94\% vs $69 \%, p=0.045)$. The overall sensitivity of the histological samples was better than for cytological samples $(82 \%$ vs $55 \%, p=0.200)$, as were accuracy $(94 \%$ vs $79 \%, p=0.101)$ and NPV $(92 \%$ vs $72 \%$, $p=0.098)$.

Specific histopathological diagnosis was obtained in 32 of $53(60.4 \%)$ PCNBs and trephine biopsies and in three of $30(10.0 \%)$ FNABs (Table 6). Three histological samples turned out to be FNs. One was a missed malignancy of the pancreas (metastasis of nephroblastoma), which turned out to be a suspected malignancy in combined MRI-guided FNAB and led to surgical excision. Two other cases were bone infections (osteomyelitis); histological samples were either normal bone or fibrosis. Five cytological samples were interpreted as FNs. One was a missed malignancy of a pancreatic lesion that turned out to be malignant in a later biopsy after mild growth. The remaining 4 cases were bone infections (osteomyelitis), where cytological samples were normal.

\section{Technical observations}

The mean lesion diameter + standard deviation (SD) was $3.8 \mathrm{~cm} \pm 4.1 \mathrm{~cm}$ (range $0.3-19 \mathrm{~cm}$ ). Five children underwent two biopsies; these were counted as individual biopsy cases.

The size of the needle tip artefact was typically $2-4 \mathrm{~mm}$.

Forty-eight of 53 (90.6\%) PCNBs or trephine biopsies were performed with one passage. When ignoring PCNBs of the soft tissue, 43 of 45 (95.6\%) bone biopsies were performed with one passage.

Forty-seven of $57(82.5 \%)$ children were ambulatory after a two- to 6-h post-biopsy rest.

Excluding nine children who needed intensive hospitalisation at the time of biopsy due to their severe clinical condition, mean hospital time in the outpatient group was 2 days (range 1-6 days, $\mathrm{SD} \pm 1.07$ days), including the biopsy day. Thirty-four of $48(70.8 \%)$ outpatients were discharged from the hospital either on the same day or 1 day

Table 4. PCNB and trephine biopsy time differences with and without technical challenges.

\begin{tabular}{lcll}
\hline & Number of biopsies & Mean needle time \pm SD (minutes) & Mean procedural time \pm SD (minutes) \\
\hline Technical challenges & 8 & $76.5 \pm 28.8$ & $93.5 \pm 30.4$ \\
Without any technical problems & 45 & $32.2 \pm 26.1$ & $47.8 \pm 32.3$
\end{tabular}

PCNB = percutaneous core needle biopsy. 
Table 5. Diagnostic performance of MRI-guided bone and soft tissue biopsies.

\begin{tabular}{lccccccccccc}
\hline & NOB & N-D & TP & TN & FP & FN & Sensitivity, Cl & Specificity, Cl & PPV, Cl & NPV, Cl & $\mathrm{DA}, \mathrm{Cl}$ \\
\hline PCNB/Trephine & 53 & 2 & 14 & 34 & 0 & 3 & $0.82[0.59-0.94]$ & $1.00[0.90-1.00]$ & $1.00[0.78-1.00]$ & $0.92[0.79-0.97]$ & $0.94[0.84-0.98]$ \\
FNAB & 30 & 6 & 6 & 13 & 0 & 5 & $0.55[0.28-0.79]$ & $1.00[0.77-1.00]$ & $1.00[0.61-1.00]$ & $0.72[0.49-0.89]$ & $0.79[0.60-0.91]$ \\
Trephine-bone & 45 & 0 & 12 & 31 & 0 & 2 & $0.86[0.60-0.96]$ & $1.00[0.89-1.00]$ & $1.00[0.76-1.00]$ & $0.94[0.80-0.98]$ & $0.96[0.85-0.99]$ \\
PCNB-soft tissue & 8 & 2 & 2 & 3 & 0 & 1 & $0.67[0.21-0.94]$ & $1.00[0.44-1.00]$ & $1.00[0.34-1.00]$ & $0.75[0.30-0.96]$ & $0.83[0.44-0.97]$ \\
FNAB-bone & 18 & 2 & 3 & 9 & 0 & 4 & $0.43[0.16-0.75]$ & $1.00[0.70-1.00]$ & $1.00[0.44-1.00]$ & $0.69[0.42-0.89]$ & $0.75[0.51-0.90]$ \\
FNAB-soft tissue & 12 & 4 & 3 & 4 & 0 & 1 & $0.75[0.30-0.95]$ & $1.00[0.51-1.00]$ & $1.00[0.44-1.00]$ & $0.80[0.38-0.97]$ & $0.88[0.53-0.98]$ \\
All & 83 & 8 & 20 & 47 & 0 & 8 & $0.71[0.53-0.85]$ & $1.00[0.92-1.00]$ & $1.00[0.84-1.00]$ & $0.85[0.74-0.93]$ & $0.89[0.80-0.94]$ \\
\hline
\end{tabular}

$\mathrm{PCNB}=$ percutaneous core needle biopsy, FNAB = fine needle aspiration biopsy, NOB = number of biopsies, N-D = non-diagnostic, TP = true positive, $\mathrm{TN}=$ true negative, $\mathrm{FP}=$ false positive, $\mathrm{FN}=$ false negative, $\mathrm{PPV}=$ positive predictive value, $\mathrm{NPV}=$ negative predictive value, $\mathrm{DA}=$ diagnostic accuracy, $\mathrm{Cl}=$ confidence interval.

Table 6. List of specific histopathological diagnoses.

\begin{tabular}{lcll}
\hline Histological samples & Number & Cytological samples & Number \\
\hline Osteomyelitis & 9 & Liver fibrosis & $\mathrm{I}$ \\
Osteoid osteoma & 9 & Spondylodiscitis & $\mathrm{I}$ \\
Sarcoma & 2 & Sarcoma & $\mathrm{I}$ \\
Enchondroma & 2 & & \\
Fibrous cortical defect & 2 & & \\
Aneurysmal bone cyst & $\mathrm{I}$ & \\
Bone cyst & $\mathrm{I}$ & \\
Osteofibrous dysplasia & $\mathrm{I}$ & \\
Spondylodiscitis & $\mathrm{I}$ & & \\
Liver fibrosis & $\mathrm{I}$ & \\
Normal bone marrow & 3 & \\
Total & 32 & \\
\hline
\end{tabular}

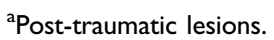

after the procedure. Forty-six of $48(95.8 \%)$ outpatients needed only anti-inflammatory drugs and paracetamol for post-biopsy pain relief.

\section{Additional biopsies}

A repeated image-guided biopsy was needed in 2 cases, a surgical open biopsy in 4 cases and a surgical extirpation in 5 cases. In two of four surgical open biopsies and in three of five surgical extirpation cases, surgery provided additional information (confirmation of diagnosis).

Four cases led to surgical open biopsy; two were soft tissue and two bone lesions. Of soft tissue lesions, one was a tumour of the nape, where both histological and cytological MRI-guided biopsy samples suggested a benign hamartomatous or cartilaginous tumour. In this case, a surgical open biopsy did not provide additional information and diagnosis was confirmed in surgical extirpation (benign hamartomatous tumour). The second was a soft tissue thickening around the periosteum in suspected osteomyelitis of a toe where a histological bone biopsy was not technically possible (bone diameter $3 \mathrm{~mm}$ ) and the MRIguided cytological sample was non-diagnostic, a surgical biopsy confirmed the diagnosis of osteomyelitis. Of bone lesions, the first was a case of osteomyelitis where diagnosis was initially reached with MRI-guided biopsy (histological sample) but faster analysed cytological sample was non-diagnostic leading to surgery where the diagnosis of osteomyelitis was confirmed. The other was a case where MRI-guided histological and cytological samples suggested a benign lesion and diagnosis was confirmed in surgery (myofibromatosis).

Two of the five lesions that led to surgical extirpation were bone biopsy cases. In both cases, MRI-guided biopsies provided diagnoses (osteoid osteoma), but extirpation was performed for therapeutic purposes. The remaining 3 cases that led to surgical extirpation were soft tissue lesions. One was a lesion of the nape, described in more detail above. One was a PCNB (MRI-guided) missed malignancy of the pancreas (metastasis of nephroblastoma), which turned out to be a suspected malignancy in FNAB (MRI-guided) and surgery confirmed malignancy. The third was a case of thickened synovia of the knee; MRI-guided histological and cytological samples were diagnostic, but operative decision led to extirpation of the lesion and confirmed the benign diagnosis.

\section{Safety}

There were no major primary complications and only two later-stage complications; a pathologic fracture appeared 7 days after femoral biopsy (a bone marrow filling enchondroma), and cellulitis 8 days after an ulnar biopsy (osteomyelitis).

\section{Discussion}

This study shows that MRI-guided biopsy is a feasible, accurate and safe guidance method in paediatric patient population to determine the nature of bone and soft tissue lesions. In our study, histological and cytological sampling was successfully performed in every case. Biopsy complications were rare. Also, the immobilisation and discharge time after the procedure was short. 
In our opinion, our results highlight the potential utility and safety of MRI guidance in performing these sometimes complex procedures in children and adolescents.

The results of our study are line with previous US-, CT and MRI-guided studies in paediatric population. ${ }^{4,7-25}$ Fritz et al. ${ }^{22}$ reported mean MRI-guided trephine biopsy length 77 min and Schulz et al. ${ }^{25}$ estimated overall MRI-guided procedural time to be roughly $60 \mathrm{~min}$. In our study, the mean procedural time of a histological biopsy without any technical challenges was shorter (48 $\mathrm{min})$. The results of this study are also in line with a previous MRI guidance study in an adult population; Kerimaa et al. ${ }^{27}$ showed a $93 \%$ accuracy, $89 \%$ sensitivity, 98\% specificity, 97\% PPV and 91\% NPV in histological bone and soft tissue biopsies. The results of our study were in concurrence to previous CTguided bone biopsy studies in adults. Leffler and $\mathrm{Chew}^{29}$ demonstrated an $82 \%$ PPV and 100\% NPV in their CTguided FNAB combined bone biopsy study. Tikkakoski et al. ${ }^{30}$ reported in their CT-guided bone biopsy study $93 \%$ diagnostic and $84 \%$ exact diagnoses rates in trephine biopsy, with the same rates in FNAB were 97\% and 90\%. However, the percentage of malignant lesions was clearly higher than in our study. In non-malignant bone lesion cases, there are few tissue cells but blood in the trephine biopsy channel where FNAB is collected; blood was the typical finding in non-diagnostic FNAB samples from bone in our study, too. Previous US- and CT-guided PCNB studies of soft tissue lesions in children show on average an $80-90 \%$ success rate for diagnosis. ${ }^{7-21}$ The soft tissue lesion results of our study are in line with previous US- and CTguided studies, although an exact histopathological diagnosis was not always achieved. The low percentage of specific histopathological diagnoses (60.4\%) of histological samples is probably explained, at least in part, by the small proportion of malignant lesions $(n=3)$, as the nature of benign lesions cannot always be specified exactly by histopathology. For example, in the case of osteomyelitis, where the histopathological finding is usually normal bone and the definitive diagnosis is based on culture analysis. Two of three patients with malignant biopsy results died during the follow-up. The remaining patient is alive after 11 years' follow-up. Biopsy complications were rare, corresponding to US- and CT-guided studies in children. ${ }^{7-21}$ Fritz et al. ${ }^{22}$ showed in their study that MRI guidance is an accurate and feasible biopsy method in children with chronic nonbacterial osteomyelitis. Their study focused on bone lesions which were visible on MRI but occult on CT. Koenig et al. ${ }^{31}$ reported that MRI has slightly better visualisation of small bone marrow lesions than CT. Ueno et al. $^{24}$ had a similar result in their study, where a scintigraphy-positive bone lesion was depicted only in MRI and a subsequent MRI-guided biopsy revealed a bone marrow metastasis. These data indicate that especially in bone marrow cases, MRI may be the best possible guidance method option. In our material, all bone marrow lesions were detected in MRI. Smith et al. ${ }^{23}$ reported in their MRIguided liver biopsy study $(n=5)$ that MRI is an accurate, feasible and safe guidance method in children and young adults. We had only one liver biopsy case in our study; the biopsy was technically successful, including exact histological and cytological diagnoses.

US is the primary image-guidance technique for soft tissue lesion biopsies. It is particularly useful in targeting superficial lesions, but has limitations, primarily acoustic shadowing from gas and bone, which can effectively prohibit biopsy in bone and lung. The use of contrast-enhanced US as a guidance tool has been limited. ${ }^{32} \mathrm{CT}$ and, historically, fluoroscopy have been the primary guiding techniques for bone biopsies. ${ }^{7,30,33}$ The superimposing normal anatomical structures and the lack of volumetric data pose a challenge to the use of fluoroscopy. CT is currently regarded as the gold standard guidance method for bone biopsy. ${ }^{7,29,30,33}$ However, modalities utilising x-rays involve radiation exposure, and the use of CT has been found to increase lifelong cancer mortality in children. ${ }^{34}$ US is inherently performed in real-time. Real-time guidance can also be achieved with CT and MRI, but it typically requires technical add-ons that can add the expenses of the procedure. Needle artefact size in MRI guidance is larger than in CT guidance. However, beam hardening artefact in CT also limits the visibility of the needle tip. In this study, the size and attributes of the instrument-generated susceptibility artefact were similar to previous reports. ${ }^{35-37}$ There were no issues with artefact asymmetry or blooming. When possible, the image readout phase was adjusted to be parallel with the biopsy needle to minimise the artefact and possible blooming effect. Regardless of the guiding modality, children's biopsies usually require general anaesthesia.

It has been shown that in some cases of musculoskeletal tumours, the use of gadolinium-contrast media may be useful in tumour targeting. ${ }^{38}$ We did not use the contrast media during biopsies, and we did not experience any significant negative effect on biopsy efficacy. However, the ability to target a viable part of the tumour can be of advantage. In the future, positron emission tomography (PET$\mathrm{CT}$ and PET-MRI) guidance may enable biopsies from the most metabolically active part of the lesion but the associated ionising radiation is a disadvantage. ${ }^{8}$

In bone biopsy cases, it seems reasonable to combine FNAB with a histological biopsy..$^{29,30}$ Often, initially nondiagnostic findings necessitate a repeated biopsy. ${ }^{39}$ In our material, the repeated image-guided biopsy did not provide additional information.

Our study is one of the few studies that provides insight and information on performing MRI-guided biopsies in a larger set of paediatric patients. This study has some important clinical and pathophysiological implications that is confirmatory to previous literature. The present research 
supports the idea that bone biopsy with MRI guidance is an alternative to $\mathrm{CT}$ guidance in paediatric population. For soft tissue targets, MRI is a useful option when US's limitations prohibit its use. CT is associated with ionising radiation and it is our view that the ALARA principle should be extended to performing paediatric biopsies, here MRI guidance could have a role. One obstacle to the generalisation of MRI use as a guidance method has been its higher costs compared to other modalities, although lower than open biopsy costs. ${ }^{40-44}$ Nevertheless, the economic arguments should not be the only consideration in utilising new medical innovations. We suggest that MRI guidance is useful as an added tool in large tertiary centres when planning the diagnostic management of paediatric patients with bone or soft tissue lesions requiring biopsy.

This study has limitations. First, the study comprises a retrospective patient series of consecutive paediatric patients without a control group. Second, the number of biopsies was still relatively small. The number of soft tissue biopsies was also limited and the number of diagnosed malignancies was low $(n=3)$. Moreover, only a few cases ended up with surgical open biopsy $(7.0 \%)$ or extirpation $(8.8 \%)$, and due to this, the direct comparison between MRI-guided biopsy and surgery obtained diagnoses was not possible in most cases.

In conclusion, MRI-guided percutaneous biopsy is a feasible, accurate and safe guidance method in paediatric cases requiring biopsy. However, further randomised and prospective studies in children comparing the accuracy of MRI-guided biopsy to that of other imaging-guided modalities are still needed.

\section{Declaration of conflicting interests}

The author(s) declared no potential conflicts of interest with respect to the research, authorship, and/or publication of this article.

\section{Funding}

The author(s) received no financial support for the research, authorship, and/or publication of this article.

\section{ORCID iD}

Mika Hirvonen (D) https://orcid.org/0000-0002-1546-0937

\section{References}

1. Nesbit ME Jr. Clinical assessment and differential diagnosis of the child with suspected cancer. In: Pizzo PA, Poplack DG (eds) Principles and practice of pediatric oncology. 2nd ed. Philadelphia, PA: Lippincott; 1993, pp. 105-114.

2. Weiss CR, Nour SG, Lewin JS. MR-guided biopsy: a review of current techniques and applications. J Magn Reson Imaging 2008; 27: 311-325.
3. Dupuy DE, Rosenberg AE, Punyaratabandhu T, et al. Accuracy of CT-guided needle biopsy of musculoskeletal neoplasms. Am J Roentgenology 1998; 171: 759-762.

4. Schulz T, Tröbs RB, Schneider JP, et al. MR Imaging-guided percutaneous procedures in children1. Acad Radiol 2005; 12: $1128-1134$.

5. Blanco RT, Ojala R, Kariniemi J, et. al. Interventional and intraoperative MRI at low field scanner - a review. Eur J Radiol 2005; 56: 130-142.

6. Blanco Sequeiros R, Ojala R, Kariniemi J, et al. MR-guided interventional procedures: a review. Acta Radiologica 2005; 46: 576-586.

7. Ghelman B. Biopsies of the musculoskeletal system. Radiologic Clin North America 1998; 36: 567-580.

8. Mitton B, Seeger LL, Eckardt MA, et al. Image-guided percutaneous core needle biopsy of musculoskeletal tumors in children. J Pediatr Hematology/Oncology 2014; 36: 337-341.

9. Sklair-Lewy M, Lebensart PD, Applbaum YH, et al. Percutaneous image-guided needle biopsy in children-summary of our experience with 57 children. Pediatr Radiol 2001; 31: 732-736.

10. Hussain HK, Kingston JE, Domizio P, et al. Imaging-guided core biopsy for the diagnosis of malignant tumors in pediatric patients. Am J Roentgenology 2001; 176: 43-47.

11. Shin H-J, Amaral JG, Armstrong D, et al. Image-guided percutaneous biopsy of musculoskeletal lesions in children. Pediatr Radiol 2007; 37: 362-369.

12. Sebire NJ and Roebuck DJ. Pathological diagnosis of paediatric tumours from image-guided needle core biopsies: a systematic review. Pediatr Radiol 2006; 36: 426-431.

13. Acord M and Shaikh R. Predictors of diagnostic success in image-guided pediatric soft-tissue biopsies. Pediatr Radiol 2015; 45: 1529-1534.

14. Metz T, Heider A, Vellody R, et al. Image-guided percutaneous core needle biopsy of soft-tissue masses in the pediatric population. Pediatr Radiol 2016; 46: 1173-1178.

15. Govender P, Jonas MM, Alomari AI, et al. Sonographyguided percutaneous liver biopsies in children. Am J Roentgenology 2013; 201: 645-650.

16. Tøndel C, Vikse BE, Bostad L, et al. Safety and complications of percutaneous kidney biopsies in 715 children and 8573 adults in Norway 1988-2010. Clin J Am Soc Nephrol 2012; 7: $1591-1597$.

17. Ilivitzki A, Abugazala M, Arkovitz M, et al. Ultrasound-guided core biopsy as the primary tool for tissue diagnosis in pediatric oncology. J Pediatr Hematology/Oncology 2014; 36: 333-336.

18. Wang H, Li F, Liu J, et al. Ultrasound-guided core needle biopsy in diagnosis of abdominal and pelvic neoplasm in pediatric patients. Pediatr Surg Int 2014; 30: 31-37.

19. Hassan SF, Mathur S, Magliaro TJ, et al. Needle core vs open biopsy for diagnosis of intermediate- and high-risk neuroblastoma in children. J Pediatr Surg 2012; 47: 1261-1266.

20. McCrone L, Alexander S, Karsli C, et al. US-guided percutaneous needle biopsy of anterior mediastinal masses in children. Pediatr Radiol 2012; 42: 40-49. 
21. Garrett KM, Fuller CE, Santana VM, et al. Percutaneous biopsy of pediatric solid tumors. Cancer 2005; 104: 644-652.

22. Fritz J, Tzaribachev N, Thomas C, et al. Magnetic resonance imaging-guided osseous biopsy in children with chronic recurrent multifocal osteomyelitis. CardioVascular Interv Radiol 2012; 35: 146-153.

23. Smith EA, Grove JJ, Van Der Spek AFL, et al. Percutaneous Magnetic-resonance-guided biopsy of focal liver lesions. Pediatr Radiol 2017; 47: 750-754.

24. Ueno S, Yokoyama S, Hirakawa H, et al. Use of real-time magnetic resonance guidance to assist bone biopsy in pediatric malignancy. Pediatrics 2002; 109: E18-E23.

25. Schulz T, Tröbs RB, Schneider JP, et al. Pediatric MR-guided interventions. Eur J Radiol 2005; 53: 57-66.

26. Blanco Sequeiros R, Klemola R, Ojala R, et al. MRI-guided trephine biopsy and fine-needle aspiration in the diagnosis of bone lesions in low-field ( $0.23 \mathrm{~T}) \mathrm{MRI}$ system using optical instrument tracking. Eur Radiol 2002; 12: 830-835.

27. Kerimaa P, Marttila A, Hyvönen P, et al. MRI-guided biopsy and fine needle aspiration biopsy (FNAB) in the diagnosis of musculoskeletal lesions. Eur J Radiol 2013; 82: $2328-2333$

28. Blanco Sequeiros R, Klemola R, Ojala R, et al. Percutaneous MR-guided discography in low-field system using optical instrument tracking: a feasibility study. J Magn Reson Imaging 2003; 17: 214-219.

29. Leffler SG and Chew FS. CT-guided percutaneous biopsy of sclerotic bone lesions: diagnostic yield and accuracy. Am J Roentgenology 1999; 172: 1389-1392.

30. Tikkakoski T, Lähde S, Puranen J, et al. Combined CT-guided biopsy and cytology in diagnosis of bony lesions. Acta Radiologica 1992; 33: 225-229.

31. Koenig CW, Duda SH, Truebenbach J, et al. MR-guided biopsy of musculoskeletal lesions in a low-field system. J Magn Reson Imaging 2001; 13: 761-768.

32. Loizides A, Widmann G, Freuis T, et al. Optimizing ultrasoundguided biopsy of musculoskeletal masses by application of an ultrasound contrast agent. Ultraschall in der Medizin (Stuttgart, Germany : 1980) 2011; 32: 307-310.

33. Trieu J, Schlicht SM and Choong PF. Diagnosing musculoskeletal tumours: how accurate is CT-guided core needle biopsy? Eur J Surg Oncol (Ejso) 2016; 42: 1049-1056.

34. Brenner DJ, Elliston CD, Hall EJ, et al. Estimated risks of radiation-induced fatal cancer from Pediatric CT. Am J Roentgenology 2001; 176: 289-296.

35. Lewin JS, Duerk JL, Jain VR, et al. Needle localization in MR-guided biopsy and aspiration: effects of field strength, sequence design, and magnetic field orientation. Am J Roentgenology 1996; 166: 1337-1345.

36. Liu H, Martin AJ and Truwit CL. Interventional MRI at highfield (1.5 T): needle artifacts. J Magn Reson Imaging 1998; 8 : 214-219.

37. Liu H, Hall WA, Martin AJ, et al. Biopsy needle tip artifact in MR-guided neurosurgery. J Magn Reson Imaging 2001; 13: 16-22.

38. Verstraete KL and Lang P. Bone and soft tissue tumors: the role of contrast agents for MR imaging. Eur J Radiol 2000; 34: 229-246.

39. Wu JS, McMahon CJ, Lozano-Calderon S, et al. JOURNAL CLUB: utility of repeat core needle biopsy of musculoskeletal lesions with initially nondiagnostic findings. Am J Roentgenology 2017; 208: 609-616.

40. Salomonowitz EK, Cejna M and Dewey C. Simple and effective technique of guided biopsy in a closed MRI system. Abdom Imaging 2000; 25: 638-642.

41. Genant JW, Vandevenne JE, Bergman AG, et al. Interventional musculoskeletal procedures performed by using MR imaging guidance with a vertically open MR unit: assessment of techniques and applicability. Radiology 2002; 223: 127-136.

42. Alanen J, Keski-Nisula L, Blanco-Sequeiros R, et al. Cost comparison analysis of low-field $(0.23 \mathrm{~T}) \mathrm{MRI}-$ and CTguided bone biopsies. Eur Radiol 2004; 14: 123-128.

43. Fraser-Hill MA, Renfrew DL and Hilsenrath PE. Percutaneous needle biopsy of musculoskeletal lesions. 2. Cost-effectiveness. Am J Roentgenology 1992; 158: 813-818.

44. Ronkainen J, Blanco Sequeiros R and Tervonen O. Cost comparison of low-field (0.23 T) MRI-guided laser ablation and surgery in the treatment of osteoid osteoma. Eur Radiol 2006; 16: 2858-2865. 